\title{
Siegel Descriptors for Image Processing
}

\author{
Reiner Lenz
}

\section{Linköping University Post Print}

\section{Tweet}

N.B.: When citing this work, cite the original article.

Reiner Lenz , Siegel Descriptors for Image Processing, 2016, IEEE Signal Processing Letters, (23), 5, 625-628.

$$
\text { http://dx.doi.org/10.1109/LSP.2016.2542850 }
$$

(C)2016 IEEE. Personal use of this material is permitted. However, permission to reprint/republish this material for advertising or promotional purposes or for creating new collective works for resale or redistribution to servers or lists, or to reuse any copyrighted component of this work in other works must be obtained from the IEEE.

\section{http://ieeexplore.ieee.org/}

Postprint available at: Linköping University Electronic Press

http://urn.kb.se/resolve?urn=urn:nbn:se:liu:diva-128143 


\title{
Siegel Descriptors for Image Processing
}

\author{
Reiner Lenz
}

\begin{abstract}
We introduce the Siegel upper half space with its symplectic geometry as a framework for low-level image processing. We characterize properties of images with the help of six parameters: two spatial coordinates, the pixel value and the three parameters of a symmetric positive-definite matrix, such as the metric tensor. We construct a mapping of these parameters into the Siegel upper half-space. From the general theory it is known that there is a distance on this space that is preserved by the symplectic transformations. The construction provides a mapping that has relatively simply transformation properties under spatial rotations and the distance values can be computed with the help of closed form expressions which allow an efficient implementation. We illustrate the properties of this geometry by considering a special case where we compute for every pixel its symplectic distance to its four spatial neighbors and we show how spatial distances, pixel value changes and texture properties are described in this unifying symplectic framework.
\end{abstract}

\section{INTRODUCTION}

Group theoretical methods provide important tools for signal and image processing. In a general setting one considers objects $x$ in some set $\mathcal{X}$ and transformations $g: \mathcal{X} \rightarrow \mathcal{X}$ where the transformations $g$ form a group $G$. In the earliest image processing applications the elements $x$ were points on a plane and the group $G$ was the group of two-dimensional rotations or translations [1]. A generalization of the same type considers groups acting on points in higher dimensional spaces. A transformation group is a very general construction and we mention a few examples relevant for signal processing: finite groups acting on grids [2], permutation groups acting on histograms [3], affine, shift-scale and general linear groups acting on subspaces [4], Lorentz groups acting on conical color spaces or acting on chromaticity spaces represented by the unit disk [5], in the context of information geometry and different imaging techniques one is studying transformation groups acting on the space of symmetric positive-definite (SPD) matrices [6] and canonical transforms describing optical systems [7]. In the following we consider the Siegel upper-half space together with the group of symplectic transformations and illustrate its usage in low-level image processing.

In the following we will use boldface symbols for vectors and matrices and use $\mathbf{x}=(x, y)$ as a two-dimensional vector with the pixel coordinates. We will use $\mathbf{0}$ for matrices and vectors where all elements are zero, the size will be clear from the context. By diag $(\mathbf{v})$ we denote the diagonal matrix with diagonal elements given by the vector $\mathbf{v}$. In the usual definition of a two-dimensional gray-valued image $I$ it is a

Copyright (c) 2015 IEEE. Personal use of this material is permitted. However, permission to use this material for any other purposes must be obtained from the IEEE by sending a request to pubs-permissions@ieee.org.

R. Lenz is with the Department of Science and Technology, Linköping University, SE-60174 Norrköping, Sweden function $I: \mathbf{x} \mapsto I(\mathbf{x})$ with $I(\mathbf{x}) \geq 0$. We will extend this definition by considering triples of the form

$$
(\mathbf{x}, \mathbf{C}(\mathbf{x}), I(\mathbf{x}))
$$

where $\mathbf{x}$ is the coordinate vector, $\mathbf{C}(\mathbf{x})$ is a symmetric, positive-definite $2 \times 2$ matrix and $I(\mathbf{x})$ is a positive scalar value. One motivation of this definition assumes that the process generating the image is a stochastic process and then $\mathrm{x}$ is the position as given by the current coordinate system, $I(\mathbf{x})$ is the mean value (or simply the intensity value at this point) and $\mathbf{C}(\mathbf{x})$ is the covariance matrix of the gray value gradient of this process. This is by now a very common construction (see Chap. 4 in [8] for an application in the context of corner detection).

From the construction we can see that a translation of the origin of the spatial coordinate system (by a translation vector $\mathbf{t}$ ) takes the simple form:

$$
(\mathbf{x}, \mathbf{C}(\mathbf{x}), I(\mathbf{x})) \mapsto(\mathbf{x}-\mathbf{t}, \mathbf{C}(\mathbf{x}-\mathbf{t}), I(\mathbf{x}-\mathbf{t})) .
$$

The transformation properties under a rotation $\mathbf{R}$ are more complicated. In the case of the gradient covariance we find that

$$
\mathbf{x} \mapsto \mathbf{R x} ; \quad \mathbf{C}(\mathbf{x}) \mapsto \mathbf{R C}(\mathbf{x}) \mathbf{R}^{\prime}
$$

where $\mathbf{R}^{\prime}$ is the transpose of $\mathbf{R}$ as usual.

In the following we will describe how this approach can be modeled in an abstract group theoretical framework. The starting point of the construction is the observation that the symmetric, positive-definite $2 \times 2$ matrices form the imaginary part of the Siegel upper half-space $\mathfrak{H}(2)$ (to be introduced later). We assume that all intensity values $I(\mathbf{x})>0$ are strictly positive. We can therefore construct a new symmetric, positive-definite $3 \times 3$ matrix which contains the original $2 \times 2$ matrix in the upper left corner and $I(\mathbf{x})$ in the lower right corner. By construction the new matrix is also symmetric and positive-definite and therefore an element of the imaginary part of the Siegel upper half-space $\mathfrak{H}(3)$. Finally we encode the position vector $\mathbf{x}$ in the real part of a point in the Siegel upper half-space and we have therefore constructed a description of $(\mathbf{x}, \mathbf{C}(\mathbf{x}), I(\mathbf{x}))$ as one point $\mathbf{Z}$ in $\mathfrak{H}(3)$.

We will then introduce the symplectic group $\operatorname{Sp}(2 n)$ as the transformation group that acts on the upper half space $\mathfrak{H}(n)$. One advantage of this construction is the fact that all tools and results from symplectic geometry can be applied to solve image processing problems. We will illustrate this with one example: distance measurements between two points $\mathbf{Z}_{1}$ and $\mathbf{Z}_{2}$ in the upper half space representing two image patches. From the general theory it is known that there is a distance measure $\mathfrak{D}\left(\mathbf{Z}_{1}, \mathbf{Z}_{2}\right)$ that is invariant under simultaneous symplectic transformation of point pairs. With the help of this distance measure we can compute the difference, or similarity 
between two points in a way that includes the positions, the intensity values and the covariance matrices at both points.

In the next section we will first collect the basic facts and results from the theory of Siegel half-spaces and symplectic geometry. We will then describe how to map the image descriptors to the upper half space and discuss some practical implementation details. Using a few examples we will illustrate some basic properties of the general theory.

\section{Siegel Upper Half Space And Symplectic GEOMETRY}

We now introduce the upper half space $\mathfrak{H}(n)$ and its group theoretically defined geometry. We will only summarize the main facts. The relevant results are derived in [9] and we will follow the notations used there. Other useful books are [10] and, from a slightly different point of view, [11]. For a description of symplectic geometry and its applications see [12].

The Siegel upper half space $\mathfrak{H}(n)$ is defined as the space of symmetric complex matrices $\mathbf{Z}=\mathbf{X}+i \mathbf{Y}$ of size $n \times n$ with positive-definite imaginary part $\mathbf{Y}$. For a matrix $\mathbf{M}$ the transpose is denoted by $\mathbf{M}^{\prime}$ and $\overline{\mathbf{Z}}$ is the conjugate complex of $\mathbf{Z}$. For a real matrix $\mathbf{M}$ and arbitrary matrix $\mathbf{L}$ we define $\mathbf{L}[\mathbf{M}]=\mathbf{M}^{\prime} \mathbf{L} \mathbf{M}$.

The geometry of the upper half-space $\mathfrak{H}(n)$ can be described with the help of the symplectic group $\operatorname{Sp}(2 n)$, defined as follows: we denote the $n \times n$ identity matrix by $\mathbf{E}$ and define the $2 n \times 2 n$ matrix $J$ as

$$
J=\left(\begin{array}{cc}
\mathbf{0} & \mathbf{E} \\
-\mathbf{E} & \mathbf{0}
\end{array}\right)
$$

Here and in the following we will use block matrix notation and the $\mathbf{0}$ in the previous definition stands thus for a $n \times n$ matrix consisting of zeros. The elements $\mathbf{M} \in \operatorname{Sp}(2 n)$ are defined as the solutions of the equation $\mathbf{J}[\mathbf{M}]=\mathbf{J}$.

Writing an element $\mathbf{M} \in \mathrm{Sp}(2 n)$ in block-matrix form we get (Eq.(7) in [9])

$$
\begin{aligned}
\mathbf{M} & =\left(\begin{array}{cc}
\mathbf{A} & \mathbf{B} \\
\mathbf{C} & \mathbf{D}
\end{array}\right) \text { with: } \\
\mathbf{A B}^{\prime} & =\mathbf{B A}^{\prime}, \mathbf{C D}^{\prime}=\mathbf{D} \mathbf{C}^{\prime}, \mathbf{A D}^{\prime}-\mathbf{B C}^{\prime}=\mathbf{E}
\end{aligned}
$$

For an element $\mathbf{M} \in \operatorname{Sp}(2 n)$ (with the usual block-matrix structure) and $\mathbf{Z} \in \mathfrak{H}(n)$ we define the symplectic map as

$$
\mathbf{M}\langle\mathbf{Z}\rangle=(\mathbf{A Z}+\mathbf{B})(\mathbf{C Z}+\mathbf{D})^{-1}
$$

This defines a one-to-one mapping of $\mathfrak{H}(n)$ onto itself and Theorem 1 in [9] states that every analytic mapping of $\mathfrak{H}(n)$ is symplectic. One can also show that $\left(\mathbf{M}_{2} \mathbf{M}_{1}\right)\langle\mathbf{Z}\rangle=$ $\mathbf{M}_{2}\left\langle\mathbf{M}_{1}\langle\mathbf{Z}\rangle\right\rangle$

Finally we introduce a metric on $\mathfrak{H}(n)$ that is invariant under symplectic maps: For any two points $\mathbf{Z}, \mathbf{Z}_{1} \in \mathfrak{H}$ define the generalization of the cross-ratio

$$
R\left(\mathbf{Z}, \mathbf{Z}_{1}\right)=\left(\mathbf{Z}-\mathbf{Z}_{1}\right)\left(\mathbf{Z}-\overline{\mathbf{Z}}_{1}\right)^{-1}\left(\overline{\mathbf{Z}}-\overline{\mathbf{Z}}_{1}\right)\left(\overline{\mathbf{Z}}-\mathbf{Z}_{1}\right)^{-1}
$$

Some important properties of the metric on $\mathfrak{H}(n)$ are (see Theorems 2-4 in [9]):
- there is a metric that is invariant under symplectic mappings

- for two arbitrary points $\mathbf{Z}, \mathbf{Z}_{1} \in \mathfrak{H}(n)$ there is exactly one geodesic arc connecting them

- for two pairs of points $\left(\mathbf{Z}, \mathbf{Z}_{1}\right)$ and $\left(\mathbf{W}, \mathbf{W}_{1}\right)$ there is a symplectic transformation that maps these two pairs into each other if and only if $R\left(\mathbf{Z}, \mathbf{Z}_{1}\right)$ and $R\left(\mathbf{W}, \mathbf{W}_{1}\right)$ have the same characteristic roots.

- denote the characteristic roots of $R\left(\mathbf{Z}, \mathbf{Z}_{1}\right)$ by $r_{\nu},(\nu=$ $1, \ldots n)$ then the distance between $\mathbf{Z}, \mathbf{Z}_{1}$ is given by ([10], p.39):

$$
\mathfrak{D}\left(\mathbf{Z}, \mathbf{Z}_{1}\right)=\sqrt{2} \cdot \sqrt{\sum_{\nu} \log ^{2} \rho_{\nu}}
$$

where we define $\rho_{\nu}=\left(1+\sqrt{r_{\nu}}\right) /\left(1-\sqrt{r_{\nu}}\right)$.

- all geodesics on $\mathfrak{H}(n)$ are symplectic images of the curves $i \operatorname{diag}\left(p_{1}^{s}, \ldots p_{n}^{s}\right)$ where $p_{\nu}$ are positive constants with $\sum_{\nu} \log ^{2} p_{\nu}=1$

\section{SIEGEL DESCRIPTORS}

We will now construct a mapping of the descriptors $(\mathbf{x}, \mathbf{C}(\mathbf{x}), I(\mathbf{x}))=(\mathbf{x}, \mathbf{C}, I)$ to a point $\mathbf{Z} \in \mathfrak{H}(3)$. We will call such a point $\mathbf{Z}$ a Siegel descriptor.

The matrix $\mathbf{C}$ is symmetric and positive definite. We also assumed that the intensity $I$ is strictly positive. We can therefore construct the matrix

$$
\mathbf{Y}(\mathbf{C}, I)=\mathbf{Y}=\left(\begin{array}{cc}
\mathbf{C} & \mathbf{0} \\
\mathbf{0} & I
\end{array}\right)
$$

For the position vector $\mathbf{x}=r(\cos \alpha, \sin \alpha)$ we define the real symmetric matrix $\mathbf{X}_{2}$ (which is essentially a combination of a reflection and a rotation):

$$
\mathbf{X}_{2}(\mathbf{x})=\mathbf{X}_{2}(r, \alpha)=\mathbf{X}_{2}=r\left(\begin{array}{cc}
\sin \alpha & \cos \alpha \\
\cos \alpha & -\sin \alpha
\end{array}\right)
$$

The Siegel descriptor is now defined as

$$
\mathbf{Z}(\mathbf{x}, \mathbf{C}, I)=\mathbf{X}(\mathbf{x})+i \mathbf{Y}(\mathbf{C}, I)
$$

with:

$$
\mathbf{X}(\mathbf{x})=\mathbf{X}=\left(\begin{array}{cc}
\mathbf{X}_{2}(\mathbf{x}) & \mathbf{0} \\
\mathbf{0} & 0
\end{array}\right)
$$

Two special types of symplectic matrices are the following:

$$
\mathbf{M}_{\mathbf{S}}=\left(\begin{array}{cc}
\mathbf{E} & \mathbf{S} \\
\mathbf{0} & \mathbf{E}
\end{array}\right) \quad \text { and } \quad \mathbf{M}_{\mathbf{Q}}=\left(\begin{array}{cc}
\mathbf{Q} & \mathbf{0} \\
\mathbf{0} & \mathbf{Q}
\end{array}\right)
$$

with symmetric $\mathbf{S}$ and rotation matrix $\mathbf{Q}$. This follows from Eq.(2) using simple calculations for the cases $\mathbf{A}=\mathbf{D}=$ $\mathbf{E}, \mathbf{C}=\mathbf{0}$ and $\mathbf{B}=\mathbf{C}=\mathbf{0}$ and $\mathbf{Q}$ a rotation matrix. The first matrix defines a (real) shift on $\mathfrak{H}(n)$ and the second matrix gives

$$
\mathbf{M}_{\mathbf{Q}}\langle\mathbf{Z}\rangle=\mathbf{Q Z Q}^{\prime}=\mathbf{Z}[\mathbf{Q}]
$$

For a block-diagonal three-dimensional rotation matrix $\mathbf{Q}$ of the form

$$
\mathbf{Q}=\left(\begin{array}{cc}
\mathbf{R} & \mathbf{0} \\
\mathbf{0} & 1
\end{array}\right)
$$


and a Siegel descriptor $\mathbf{Z}(\mathbf{x}, \mathbf{C}, I)$ we see that $\mathbf{M}_{\mathbf{Q}}$ leaves $I$ untouched and maps $\mathbf{C}$ to $\mathbf{C}[\mathbf{R}]$ as desired. For the real part we find however that

$$
\mathbf{X}_{2}(r, \alpha) \mapsto \mathbf{X}_{2}(r, \alpha-2 \beta)
$$

where $\beta$ is the rotation angle characterizing $\mathbf{R}$. Instead of the simple rotation $\mathbf{x} \mapsto \mathbf{R} \mathbf{x}$ we get thus a rotation with a double angle $2 \beta$.

For the distance between two Siegel descriptors on can see that it is essentially an independent combination of the distance in the two-parameter Siegel upper half space (defined by $\mathbf{x}$ and $\mathbf{C}$ ) and the logarithmic distance in the third (intensity) parameter. For two Siegel descriptors

$$
\mathbf{W}_{1}=\left(\begin{array}{cc}
\mathbf{Z}_{1} & \mathbf{0} \\
\mathbf{0} & I_{1}
\end{array}\right) \quad \text { and } \quad \mathbf{W}_{2}=\left(\begin{array}{cc}
\mathbf{Z}_{2} & \mathbf{0} \\
\mathbf{0} & I_{2}
\end{array}\right)
$$

we find

$$
\mathfrak{D}\left(\mathbf{W}_{1}, \mathbf{W}_{2}\right)=\sqrt{2} \cdot \sqrt{\log ^{2} \rho_{1}+\log ^{2} \rho_{2}+\log ^{2} \frac{I_{1}}{I_{2}}}
$$

where the first two terms are computed from the two-parameter case (see Eq.(3)). The fact that the distance depends only on the ratio between the two intensity values follows also from the observation that the symplectic matrices with zero blocks $\mathbf{B}=$ $\mathbf{C}=\mathbf{0}$ and diagonal matrix $\mathbf{A}=\operatorname{diag}(1,1, \gamma)$ act as scaling with factor $\gamma$ on the intensity part of the Siegel descriptor. Since the distance is invariant to simultaneous scalings of two descriptors we find that the distance depends only on the ratio.

\section{IMPLEMENTATION AND ILLUSTRATIONS}

This definition of the Siegel descriptors has the advantage that eigenvalue computations are only necessary for $2 \times 2$ matrices for which closed form expressions are available. All numerical computations, like the cross-ratio and eigenvalue computations, can be parallelized. In our experiments the two positivity conditions are enforced as follows: at points $\mathrm{x}$ for which $I(\mathbf{x})$ is exactly zero we replace $I$ with the minimum non-zero value over the whole image. For singular matrices $\mathbf{C}(\mathbf{x})$ we replace it with the nearest, already computed nonsingular $\mathbf{C}$.

The definition of the Siegel descriptors involves two, more or less arbitrary, scaling factors $c_{\mathbf{X}}, c_{\mathbf{Y}}$ since we could also use: $\mathbf{x} \mapsto c_{\mathbf{X}} \mathbf{X}$ and $(\mathbf{C}, I) \mapsto c_{\mathbf{Y}} \mathbf{Y}$ in the construction of the descriptors. Scaling the intensity part has no effect on the distance computations since only ratios of intensities are used. In our implementation we use always a weight factor of one for the part depending on $\mathbf{C}$ and use weight factors $c_{\mathbf{x}}$ and $c_{I}$ to control the influence of the other two descriptors relative to the matrix descriptor.

Apart from these constants one has to choose the pair of gradient filters and the region over which the covariance matrix and the mean intensity values are computed. In our experiments we choose the Sobel filters and quadratic regions around the pixel of interest. In the illustrations we use squares of side-lengths five and 19.

The following experiments consist of two steps: first we map all pixels to Siegel descriptors. For a descriptor $\mathbf{Z}$ we then select its four nearest descriptors $\mathbf{W}_{k}, k=1, \ldots, 4$ and

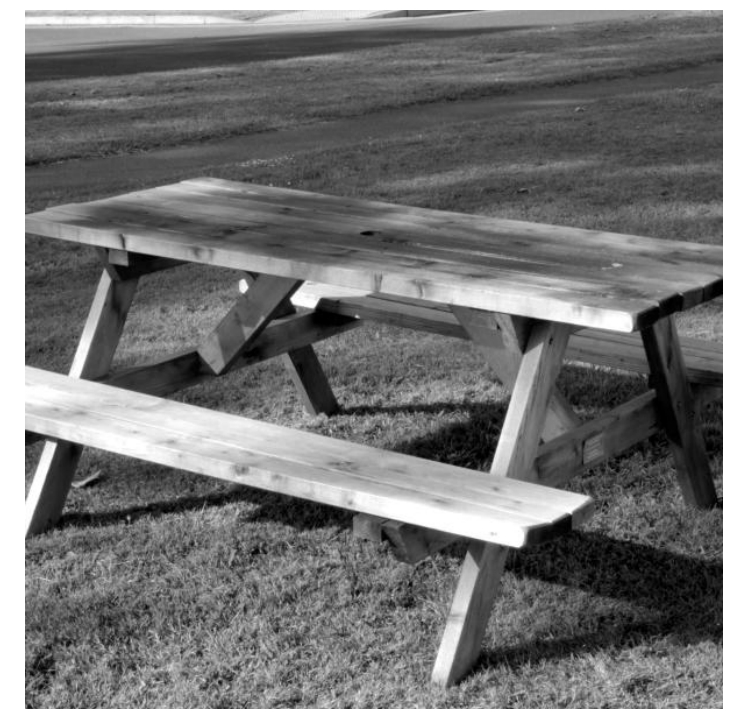

Fig. 1. Original Image

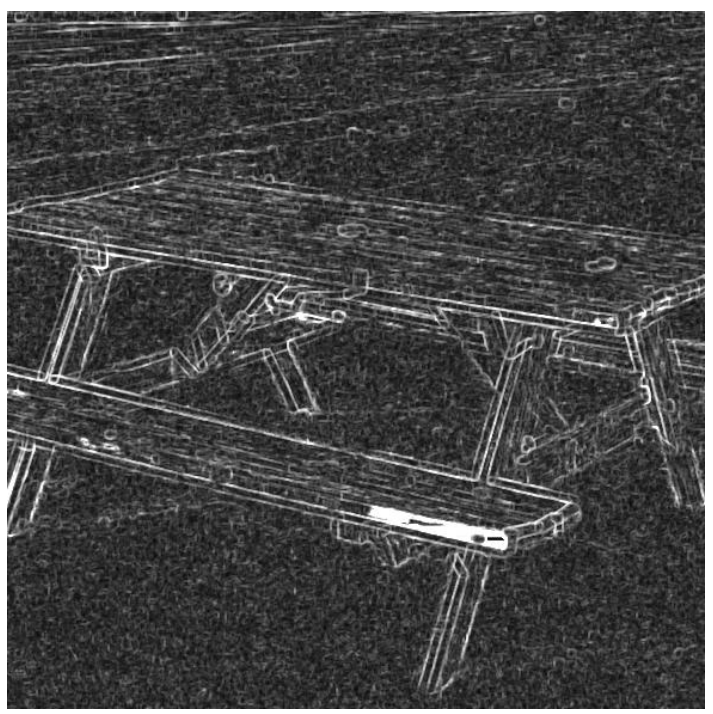

Fig. 2. Region size $5, c_{\mathbf{x}}=c_{I}=0$

compute the distance values $d_{k}=\mathfrak{D}\left(\mathbf{Z}, \mathbf{W}_{k}\right)$. The final distance value is then computed as the Euclidean length of this distance vector $d=\|(d 1, d 2, d 3, d 4)\|$. Images of these distance images are shown in the following figures. As a last comment regarding these figures we mention that very homogeneous or very black regions may produce very large distance values $d$. We therefore truncated the distance values with the value of the 0.995 quantile. These truncated and normalized images are shown in the result images.

Figure 1 shows the original image which is of size $600 \times 600$ pixels with 16 bits/pixel gray value (available at http://testimages.tecnick.com and described in [13]). For an averaging window of size $5 \times 5$ and constants $c_{\mathbf{x}}=c_{I}=0$ representing the $\mathbf{C}$ part only we obtain the image shown in Fig. 2. The selection of the constants $c_{\mathbf{x}}, c_{I}$ depends on the usage of the distance measure in later processing steps. Here we selected very high values (chosen based on the statistical properties of the eigenvalues and the gray values) to illustrate 


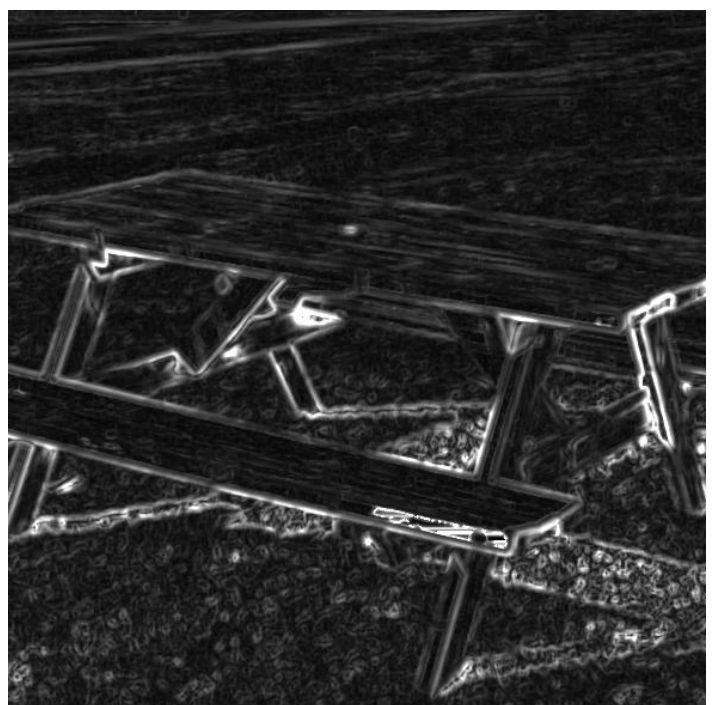

Fig. 3. Region size $5, c_{\mathbf{x}}=0, c_{I}=500$

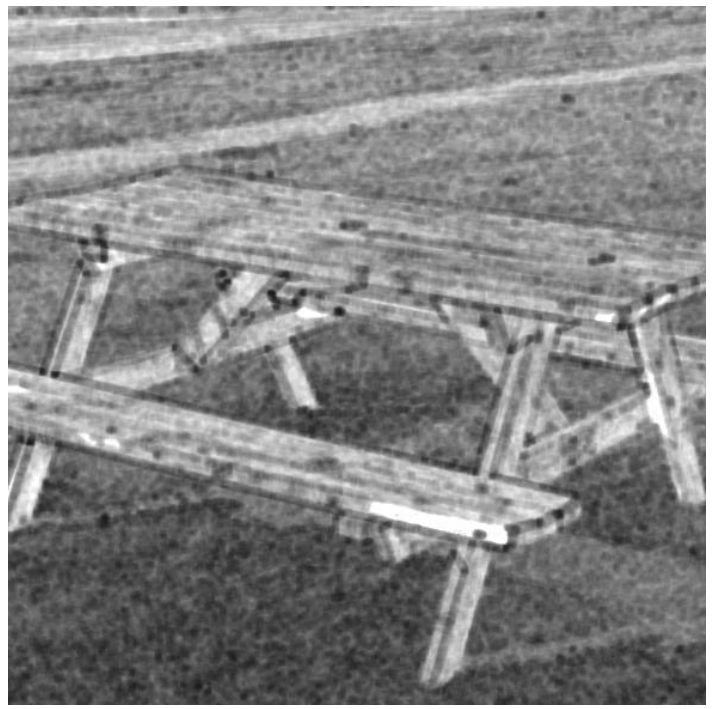

Fig. 4. Region size $5, c_{\mathbf{x}}=500, c_{I}=0$

the effect of these constants. In Fig. 3 we used $c_{\mathbf{x}}=0, c_{I}=$ 500 . The large value of the intensity weight leads to distances that mainly represent the gray value differences between neighboring pixels. In Fig. 4 we used $c_{\mathrm{x}}=500, c_{I}=0$ illustrating the influence of the position variable. Compared with the previous results one can see that in this image the path in the top of the image is clearly visible as a light band.

In the final example, Fig. 5, we use $c_{\mathbf{x}}=500, c_{I}=500$ and a large square with $19 \times 19$ pixels for the statistical computations. We see that the overall impression of the result is similar to the one obtained with parameters $c_{\mathbf{x}}=500, c_{I}=0$ and that the image is blurred because of the large region size.

\section{SUMMARY AND CONCLUSIONS}

We introduced the symplectic geometry of the Siegel upper half space as a tool with which basic properties of an image, like the location of pixels, gray values and the correlations

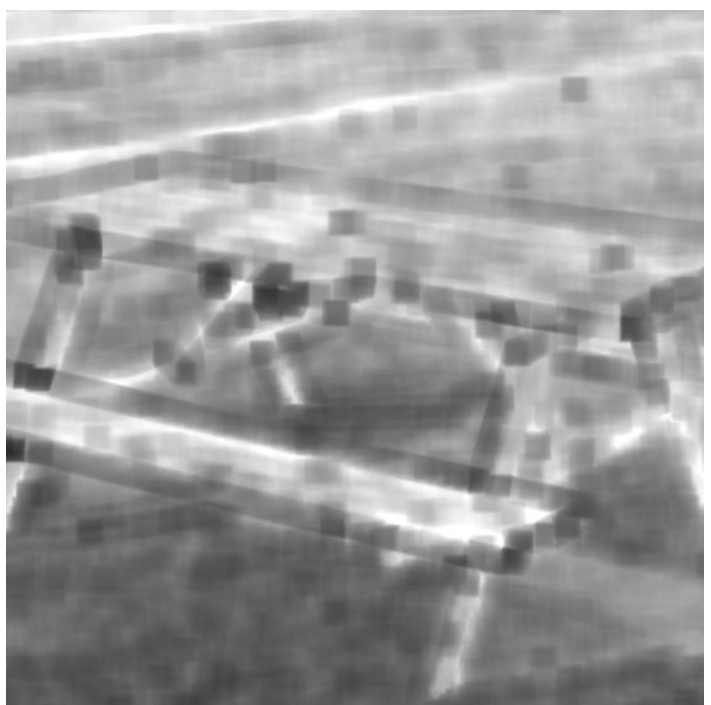

Fig. 5. Region size $19, c_{\mathbf{x}}=500, c_{I}=500$

between pixels can be investigated in a unified framework. We constructed a mapping which includes both PCA-like analysis of the covariance matrix, simple transformation rules of the position part and which leads automatically to a logarithmic description of the intensities which also characterizes perception as modeled in the Weber Fechner law. Here we only described one application based on the existence of an invariant distance on this space but considering the fundamental role of symplectic geometry in theoretical physics and mathematics we expect that many other applications in signal processing can be found.

\section{REFERENCES}

[1] R. Lenz, Group Theoretical Methods in Image Processing. New York, NY, USA: Springer-Verlag New York, Inc., 1990.

[2] R. Lenz, V. Zografos, and M. Solli, "Dihedral color filtering," in Advanced Color Image Processing and Analysis. Springer, 2013, pp. 119-145.

[3] R. Lenz and P. L. Carmona, "Hierarchical S(3)-coding of RGB histograms," in Computer Vision, Imaging and Computer Graphics. Theory and Applications. Springer, 2010, pp. 188-200.

[4] Y. Chikuse, Statistics on special manifolds. Springer Science \& Business Media, 2012, vol. 174.

[5] R. Lenz, "Spectral color spaces: Their structure and transformations," Advances in imaging and electron physics, vol. 138, pp. 1-67, 2005.

[6] F. Barbaresco, "Information geometry of covariance matrix: CartanSiegel homogeneous bounded domains, Mostow/Berger fibration and Fréchet median," in Matrix Information Geometry, F. Nielsen and R. Bhatia, Eds. Springer Berlin Heidelberg, 2013, pp. 199-255.

[7] J. S. Mondragón and K. B. Wolf, Eds., Lie methods in optics. Springer, 1986.

[8] R. Szeliski, Computer Vision: Algorithms and Applications. Springer London, 2010.

[9] C. L. Siegel, "Symplectic geometry," American Journal of Mathematics, vol. 65 , no. 1, pp. 1-86, 1943.

[10] H. Maass, Siegel's modular forms and Dirichlet series, ser. Lecture Notes in Mathematics. Springer, 1971, vol. 216.

[11] A. Terras, Harmonic analysis on symmetric spaces and applications II. Springer Science \& Business Media, 2012.

[12] A. Fomenko, Symplectic Geometry. Taylor \& Francis, 1995.

[13] N. Asuni and A. Giachetti, "Testimages: a large-scale archive for testing visual devices and basic image processing algorithms." in Eurographics Italian Chapter Conference, 2014, pp. 63-70. 